
September 2018, Pisa, Italy

\title{
Investigation of a passive control system for limiting cavitation inside turbomachinery under different operating conditions
}

\author{
T. Capurso ${ }^{\mathrm{a}, *}$, G. Menchise ${ }^{\mathrm{a}}$, G. Caramia ${ }^{\mathrm{a}}$, S.M. Camporeale ${ }^{\mathrm{a}}$, B. Fortunato ${ }^{\mathrm{a}}$, M. Torresi ${ }^{\mathrm{a}}$ \\ ${ }^{a}$ Department of Mechanics, Mathematics and Management, Polytechnic University of Bari, Via Amendola 126/B, Bari 70125, Italy
}

\begin{abstract}
Herein, a new passive control system for limiting cavitation inside turbomachinery has been applied to a NACA0009 hydrofoil. The basic idea is to introduce slots nearby its leading edge, connecting pressure and suction sides of the hydrofoil, in order to increase locally the pressure on its suction side and to prevent cavitation from developing. The cavitating flow developed around a two-dimensional hydrofoil is here considered since it is an archetype of cavitation nearby the leading edges of the impeller vanes. Thus, the flow field developed at the leading edge of both the original and modified hydrofoil has been studied at different angles of attack in order to reproduce a wide range of operating conditions that occur inside turbomachinery. Eventually, a comparison of their performance in terms of polars $\left(C_{L}\right.$ and $\left.C_{D}\right)$ and vapour volume fractions $\left(\alpha_{v}\right)$ is performed.
\end{abstract}

(C) 2018 The Authors. Published by Elsevier Ltd.

This is an open access article under the CC BY-NC-ND license (https://creativecommons.org/licenses/by-nc-nd/4.0/)

Selection and peer-review under responsibility of the scientific committee of the 73rd Conference of the Italian Thermal Machines Engineering Association (ATI 2018).

Keywords: Cavitation; Multi-phase flow; OpenFOAM; Passive cavitating flow control system; Centrifugal pump

\section{Introduction}

Cavitation has an important impact on the reliability of hydraulic systems and can lead to a reduction of performance or worse to a total machine failure. Thus, it is important to understand what causes cavitation and how it can be avoided in order to improve operating range and reliability. Cavitation is a phenomenon, which can occur in hydraulic turbomachineries, such as pumps, turbines and propellers, when the local static pressure falls below the vapour pressure. The phase change is characterized by pressure oscillations with specific amplitudes and frequencies. Cavitation also causes surface damages and induces noise and vibrations, which negatively affect the performance of these machines [1,2]. Several experiments have been performed to study cavitation around 2D hydrofoils since they can be representative, for instance, of the fluid flow around impeller vanes [3, 4]. Furthermore, different numerical algorithms have been developed with the aim of simulating cavitating flow around Venturi duct and 2D hydrofoils

\footnotetext{
${ }^{*}$ Corresponding author. Tel.: +39-327-915-035-2.

E-mail address: tommaso.capurso@poliba.it
} 
$[5,6]$ and various turbulence models have been tested in order to find the best combination between mass transfer models and turbulence models [7].

In the past, either active or passive cavitating flow control systems have been proposed with the aim of limiting cavitation phenomena inside turbomachines and around hydrofoils. The formers are intentionally activated by the operator like in the case of ventilation where gas is injected and a cavity is generated around the body in order to reduce vapour shedding and bubble collapse [8]. The latters are systems which work autonomously with the aim of modifying the boundary layer behaviour. In this second class, we can find the application of external elements, e.g., trip bars installed along the bodies [9, 10]. Actually, our cavitation control system belongs to this second class. In particular, the flow past a NACA0009 hydrofoil at a fixed angle of attack equal to $2.5^{\circ}$ was investigated by means of OpenFOAM and the results compared against experimental data provided by P. Dupont [11]. Furthermore, a modified NACA0009 hydrofoil with slots, connecting the pressure side to the suction side of the foil, introduced as a passive cavitation control system, was investigated in order to understand the influence on the performance under fully wetted flow and when cavitation occurs [12]. Furthermore, the capability of the ducts to limit flow separation on the suction side of the hydrofoil was pointed out.

In this work we intend to investigate the NACA0009 at different angles of attack representing a hydraulic turbomachinery when operated under either design $\left(0^{\circ}\right)$ or off design $\left(2.5^{\circ}, 5^{\circ}\right)$ conditions. The same simulations have been run with the application of the passive system control and eventually a comparison of their performance in terms of force coefficients $\left(C_{L}\right.$ and $\left.C_{D}\right)$ and vapour volume fractions $\left(\alpha_{v}\right)$ is performed. Simulations have been performed by means of the open source CFD code OpenFOAM by solving the 2D Unsteady Reynolds-Average Navier-Stokes (U-RANS) equations for incompressible flow with the mass transfer model proposed by Schnerr and Sauer [13]. The attached cavities are strongly connected to the boundary layer behavior, while the bubble cavitation chiefly depends on the pressure distribution and the liquid nuclei content [15]. Because of the small angles of attack $\left(\max 5^{\circ}\right)$ this activity deals with quasi-steady cavitation and periodic cavitation. These phenomena require a transient approach. Even if high Reynolds numbers are considered, laminar to turbulent transition is crucial in order to capture the inception of attached cavitation, which occurs in the small recirculating bubble following laminar separation of the boundary layer, as it does for the transcritical flow around a circular cylinder [15]. Hence, a transition-sensitive turbulence closure model can help in predicting the transition phenomena. For this reason the $k-k l-\omega[14,15]$ model has been used coherently with Capurso et al. [7]. The passive cavitation control system shows a remarkable reduction of the vapour volume fraction (from $-93 \%$ to $-54 \%$ ), however accompanied by a loss of performance corresponding to a reduction of $C_{L} / C_{D}$ from $-39 \%$ to $-45 \%$ at $\alpha=2.5^{\circ}$ and $5^{\circ}$, respectively.

\section{Nomenclature}

$$
\begin{array}{ll}
\rho & \text { density }\left[\mathrm{kg} / \mathrm{m}^{3}\right] \\
\mu & \text { viscosity }[\mathrm{kg} / \mathrm{ms}] \\
p & \text { pressure }[\mathrm{Pa}] \\
C_{v} & \text { evaporation coefficient } \\
C_{c} & \text { condensation coefficient } \\
\dot{m}^{-} & \text {evaporation rate } \\
\dot{m}^{+} & \text {condensation rate } \\
\sigma & \text { cavitation number } \\
p_{v} & \text { saturation pressure }[\mathrm{Pa}] \\
C_{p} & \text { pressure coefficient } \\
C_{L} & \text { lift coefficient } \\
C_{D} & \text { drag coefficient } \\
L & \text { lift per unit lenght }[\mathrm{N} / \mathrm{m}] \\
D & \text { drag per unit lenght }[\mathrm{N} / \mathrm{m}] \\
\alpha_{v} & \text { vapour volume fraction } \\
\alpha & \text { angle of attack }
\end{array}
$$




\section{Governing equations}

The Unsteady RANS equations in their conservative unsteady formulation, for Newtonian fluids without body forces and heat transfer, are presented in the Cartesian coordinates (1) along with the continuity equation (2) and the mass transport equation (3) for liquid:

$$
\begin{aligned}
& \frac{\partial \rho_{m} u_{i}}{\partial t}+\frac{\partial\left(\rho_{m} u_{j} u_{i}\right)}{\partial x_{j}}=-\frac{\partial p}{\partial x_{j}}+\frac{\partial}{\partial x_{j}}\left[\mu_{m}\left(\frac{\partial u_{i}}{\partial x_{j}}+\frac{\partial u_{j}}{\partial x_{i}}\right)-\mu_{m} \frac{2}{3} \frac{\partial u_{k}}{\partial x_{k}} \delta_{i j}\right] \\
& \frac{\partial \rho_{m}}{\partial t}+\frac{\partial\left(\rho_{m} u_{j}\right)}{\partial x_{j}}=0 \\
& \frac{\partial\left(\rho_{l} \alpha_{l}\right)}{\partial t}+\frac{\partial\left(\rho_{l} \alpha_{l} u_{j}\right)}{\partial x_{j}}=\dot{m}^{+}+\dot{m}^{-} \\
& \rho_{m}=\rho_{l} \alpha_{l}+\rho_{v} \alpha_{v} \\
& \mu_{m}=\mu_{l} \alpha_{l}+\mu_{v} \alpha_{v}
\end{aligned}
$$

The subscript $(l, v, m)$ refers to the liquid, vapour and mixture phases, respectively. The subscripts $(i, j, k)$ denote the directions of the Cartesian coordinates. The source term, $\left(\dot{m}^{+}\right)$, and the sink term, $\left(\dot{m}^{-}\right)$, in equation (3) represent the condensation and evaporation rates, respectively. In OpenFOAM, the application, which allows one to solve this set of equations, is named InterPhaseChangeFoam. It is based on the Volume of Fluid (VoF) technique, which considers two incompressible, isothermal and immiscible flows and solves one global momentum equation and a transport equation for each flow adopting a PIMPLE algorithm. In these simulations the condensation and the evaporation rates are modeled according to Schnerr and Sauer [11]:

$$
\begin{aligned}
& \dot{m}^{-}=3 C_{v} \frac{\rho_{v} \rho_{l}}{\rho_{m}} \sqrt{\frac{2}{3 \rho_{l}}} \frac{1}{R_{B} \sqrt{\left|p-p_{v}\right|}}\left(1-\alpha_{n u c}+\alpha_{l}\right) \min \left(p-p_{v}, 0\right) \\
& \dot{m}^{+}=3 C_{c} \frac{\rho_{v} \rho_{l}}{\rho_{m}} \sqrt{\frac{2}{3 \rho_{l}}} \frac{1}{R_{B} \sqrt{\left|p-p_{v}\right|}} \alpha_{l} \max \left(p-p_{v}, 0\right)
\end{aligned}
$$

which are based on the Rayleigh-Plesset equation. The volume fraction of the water vapour nuclei, $\alpha_{n u c}$, and the radius of the bubble, $R_{B}$, used in the previous equations $(6,7)$ are calculated as follows:

$$
\begin{aligned}
\alpha_{n u c} & =\frac{n_{0} \pi d_{n u c}^{3} / 6}{1+n_{0} \pi d_{n u c}^{3} / 6} \\
R_{B} & =\sqrt[3]{\frac{3}{4 \pi n_{0}} \frac{1+\alpha_{n u c}-\alpha_{l}}{\alpha_{l}}}
\end{aligned}
$$

For these simulations $n_{0}$ and $d_{n u c}$ are set equal to $1.6 \cdot 10^{13} 1 / \mathrm{m}^{3}$ and $2 \cdot 10^{-6} \mathrm{~m}$, respectively. Actually, these values can be determined during water quality experimental tests. Both the condensation coefficient, $C_{c}$, and the evaporation coefficient, $C_{v}$, coincide to 1 , the default values in OpenFOAM.

The Schnerr and Sauer mass transfer model equations have been solved in combination with the $k-k l-\omega$ model for turbulence closure [7, 14]. The gradient terms have been computed using a second-order upwind scheme and a cell limited scheme for the pressure. The convection terms have been computed using a second-order backward scheme and a first-order upwind scheme for the turbulence terms. The Laplacian terms have been resolved using a limited corrected scheme while the time integration scheme is a first-order Euler one. 


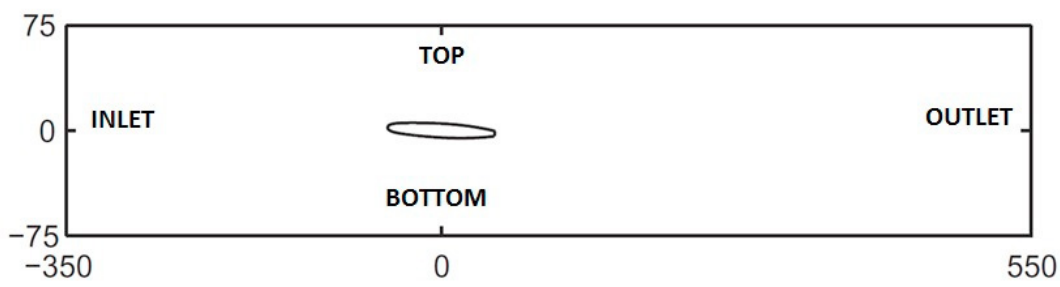

Fig. 1. Main dimensions of the numerical domain expressed in $\mathrm{mm}$.

\section{Numerical domain and boundary conditions}

The 2D fluid domain is shown in Figure 1. It reproduces the height of the experimental test section of the Cavitation Research Facility at EPFL, Lausanne [11], whereas the computational domain extends $3.5 c$ upstream and $5.5 c$ downstream of the foil $(c=100 \mathrm{~mm})$ to reduce influence of boundary conditions at the inlet and outlet.

The hydrofoil is a NACA0009 $[11,16]$ with a chord truncated at $90 \%$ of its initial chord value $(110 \mathrm{~mm})$, maximum thickness at $50 \%$ of the initial chord and the trailing edge height equal to $3.22 \mathrm{~mm}$ (Figure 2). A no-slip boundary condition is imposed along the hydrofoil surface and along the top and bottom tunnel boundaries. The pressure at the outlet is set in such a way to guarantee the desired cavitation number, $\sigma=\left(p_{\infty}-p_{v}\right) /\left(0.5 \rho_{\infty} U_{\infty}^{2}\right)=0.81$, where $p_{\infty}$ is the pressure measured at $250 \mathrm{~mm}$ upstream of the foil. This distance has been chosen being adequately far from the pressure disturbance due to the hydrofoil. The velocity at the inlet, $U_{\infty}$, is equal to $20.7 \mathrm{~m} / \mathrm{s}$ and the properties of the fluid are assumed to be $\rho_{l}=998.7 \mathrm{~kg} / \mathrm{m}^{3}$ and $v_{l}=1.08 \cdot 10^{-6} \mathrm{~m}^{2} / \mathrm{s}$, which corresponds to pure water at 17 ${ }^{\circ} \mathrm{C}$. The vapour pressure of the water at $17{ }^{\circ} \mathrm{C}$ is $p_{v}=1938.4 \mathrm{~Pa}$ and the vapour density and kinematic viscosity are $\rho_{v}=0.014493 \mathrm{~kg} / \mathrm{m}^{3}$ and $v_{v}=6.66 \cdot 10^{-4} \mathrm{~m}^{2} / \mathrm{s}$, respectively [11]. Furthermore, for this test case a constant turbulent intensity has been assumed equal to $1 \%$ at the inlet of the domain. The grid has been computed with the software Pointwise Gridgen ${ }^{\circledR}$, exported in .msh format and then converted with the command fluentMeshToFoam in OpenFOAM. After that, the grid has been checked, to extract information about its extreme values of aspect ratio (AR $<1000)$, skewness $\left(S_{k}<0.98\right)$ and non-orthogonal cells $\left(\theta_{o}<70^{\circ}\right)$, with the command checkMesh. About the grid sensitivity analysis, this has been carried out by considering the three grids having different numbers of prism layers and initial heights: a fine, a medium and a coarse mesh that are made up of 65000,38000 and 28000 cells, respectively. These grids have been tested under steady-state fully wetted (non-cavitating) condition. The details of the grid are shown in Figure 2. Each fluid mesh has been made with a different number of structured elements close to the foil surface to ensure a proper value of $y^{+}=y U_{\tau} / v$, where $y$ is the first cell height, and $U_{\tau}$ is the wall frictional velocity. The region outside these elements has been discretized with unstructured triangular elements. Mesh refinements are performed both at leading and trailing edges of the foil (Figure 2). The lift and drag coefficients have been compared with the experimental data provided by P. Dupont [11] in Table 1.

Table 1. Comparison of the hydrofoil performance (lift and drag coefficient) in wetted simulation with $U=20 \mathrm{~m} / \mathrm{s}$ compared with experimental data [11].

\begin{tabular}{|c|c|c|c|c|c|}
\hline An example of a column heading & n. cells & $C_{D}=\frac{D}{1 / 2 \rho U^{2} c}$ & $C_{L}=\frac{L}{1 / 2 \rho U^{2} c}$ & err. $C_{D}$ & err. $C_{L}$ \\
\hline Dupont P. (experimental) & & 0.0214 & 0.315 & & \\
\hline Coarse mesh $\left(y^{+}=70\right)$ & 28000 & 0.0162 & 0.334 & $-24 \%$ & $6 \%$ \\
\hline Medium mesh $\left(y^{+}=30\right)$ & 38000 & 0.0134 & 0.322 & $-49 \%$ & $2 \%$ \\
\hline Fine mesh $\left(y^{+}=1\right)$ & 65000 & 0.0207 & 0.341 & $-5 \%$ & $8 \%$ \\
\hline
\end{tabular}

The effect of turbulence models, mesh types, and mesh density on the cavitating flow dynamics have been studied in detail for the case of the NACA0009 hydrofoil at a fixed angle of attack, $\alpha=2.5^{\circ}$, under steady flow conditions for the quasi-steady sheet cavitating case $\left(\sigma=0.81\right.$ and $\left.R e=2 \cdot 10^{6}\right)$. The mesh with about 65000 cells has been selected to carry out the multi-phase analyses considering that only marginal deviations are observed in the results among the 


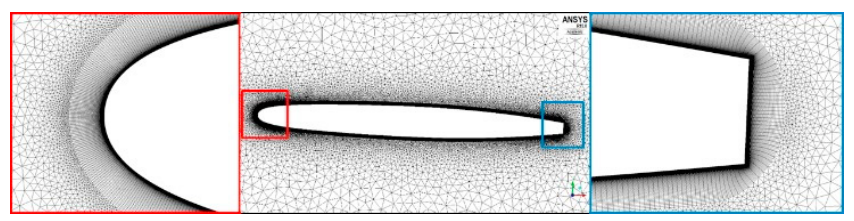

Fig. 2. Mesh details.

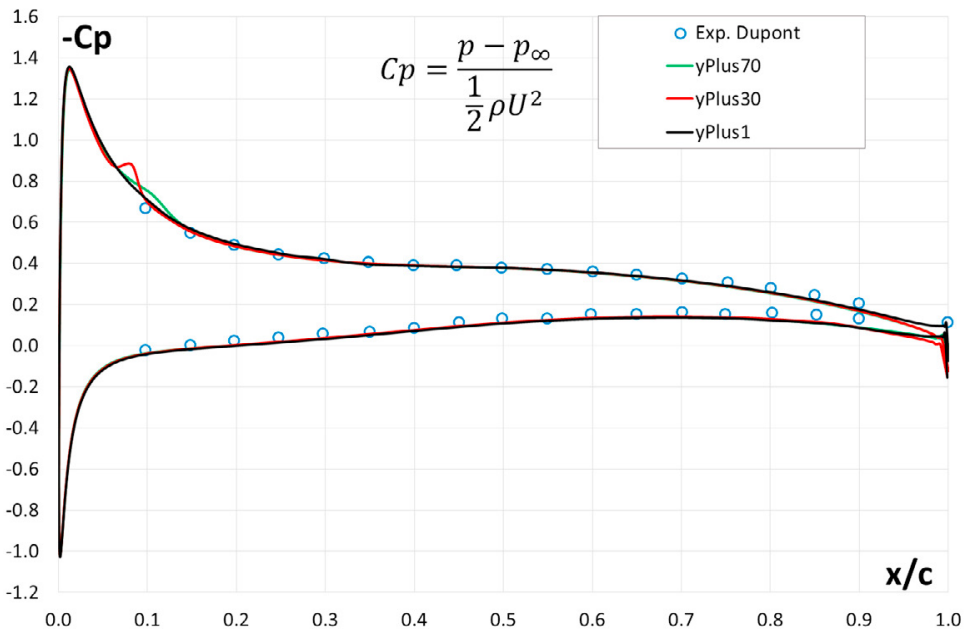

Fig. 3. Comparison of the pressure coefficient calculated with three different grids on the suction and pressure side of the NACA0009 hydrofoil with $\alpha=2.5^{\circ}$ in fully wetted flow condition with $k-k l-\omega$ turbulence model. The results are compared with experimental data [11].

three grids (see Figure 3), but this grid provides the lowest error in terms of lift and drag coefficient with respect to the experimental data. Moreover, the grid with 65000 cells presents a better resolution near the walls and the leading edge (with $y^{+}$value approximately equal to 1 ) than the mesh with 38000 cells.

\section{Passive control system}

Herein, a new passive control system for limiting cavitation has been applied to a NACA0009 hydrofoil. The basic idea is to introduce slots nearby its leading edge connecting pressure and suction sides of the hydrofoil, in order to increase locally the pressure on its suction side. The regular geometry has been modified with the introduction of three slots close to the leading edge of the foil (see Figure 4). The slots have variable cross section areas and their axes are inclined $30^{\circ}$ with respect to the chord line of the foil. Due to the pressure gradient, a small amount of flow rate flows through the slots. The slots angle is designed in order to avoid flow separation at their outlets [12]. In addition, the main flow pushes down the flow coming from the slots. An evident result is a higher boundary layer close to the suction side of the foil. The slots can be broached with a specific tool. Their cross section areas have been chosen not constant (they increase from pressure to suction side) in order to reduce the velocity of the flow toward the outlet of the slots. This slots configuration is applied to NACA0009 hydrofoil with three different angles of attack $\left(0^{\circ}, 2.5^{\circ}\right.$ and $5^{\circ}$ ) trying to reproduce the operating condition of a centrifugal pump at part-load. The NACA0009, modified with the introduction of the slots from pressure to suction side, has been discretized by using the same technique previously described. Moreover, the regions inside the slots have been discretized with structured elements close to the walls in order to guarantee the same first cell height as the one applied on the adjacent walls of the foil, see Figure 4. 

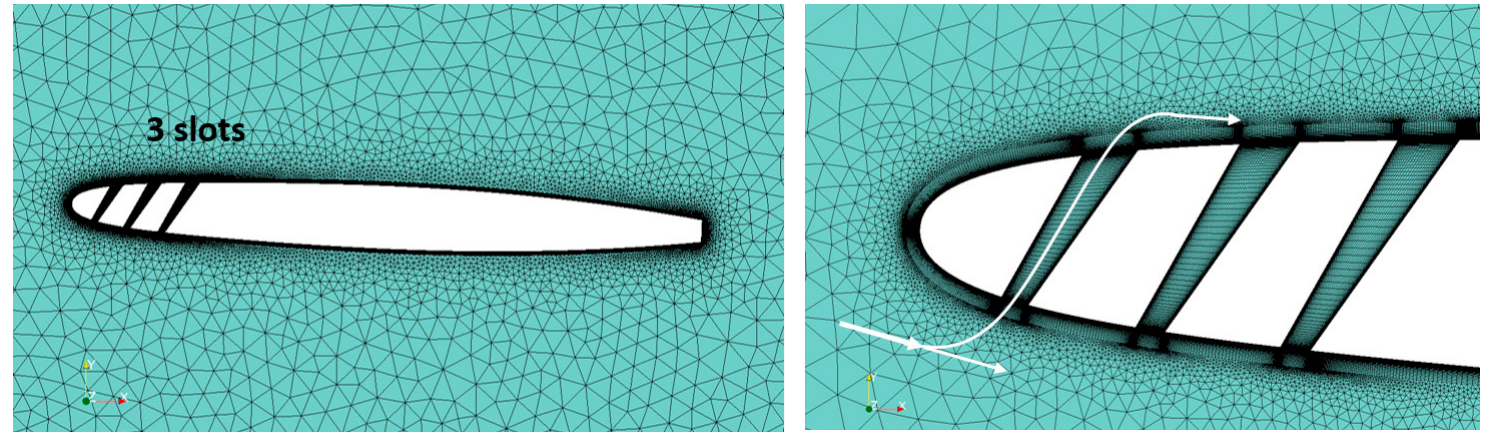

Fig. 4. NACA0009 hydrofoil modified with the introduction of the slots and a representation of the fluid flowing through the slots from the pressure to the suction side.

\section{Results and Discussion}

The unsteady simulations in both wetted and cavitating flow conditions have been run with a $\Delta t=5.0 \cdot 10^{-6} \mathrm{~s}=$ $t_{\infty} / 1000=\left(c / U_{\infty}\right) / 1000$ for a total time equal to $0.35 \mathrm{~s}$, which corresponds to seventy passages of the flow over the hydrofoil. This value of the time-step, $\Delta t$, has been selected to guarantee numerical stability. The Courant number for all the simulations is approximately the same and it is equal to 0.3. Eventually, the results have been averaged over the time interval corresponding to ten times the reference time $\left(10 t_{\infty}\right)$.

\subsection{Single-phase flow}

After the numerical method assessment, the regular foil and the modified geometry have been investigated. A comparison of the pressure coefficient obtained via numerical simulations for the regular geometry and the modified one with $\alpha=2.5^{\circ}$ is shown in Figure 5a. They are compared with the experimental data provided by P. Dupont [11]. The curves depicted in Figure 5a point out that the passive cavitation control system, acting on the forward part of the hydrofoil, increases the pressure located at the leading edge $\left(-C_{p}\right.$ decreases) and modifies the pressure distribution up to the $30 \%$ of the chord. Furthermore, the geometry modifications and the new pressure field lead to a reduction of the lift coefficient $(-25 \%)$ and an increase of the drag coefficient $(+39 \%)$.

a

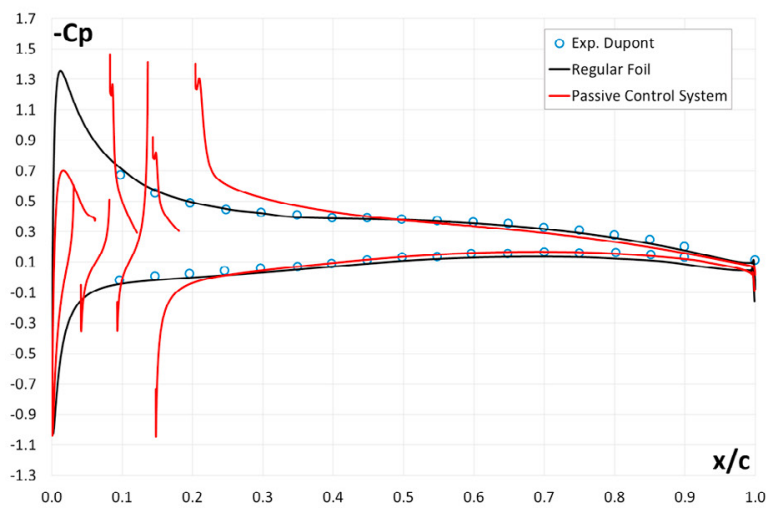

b

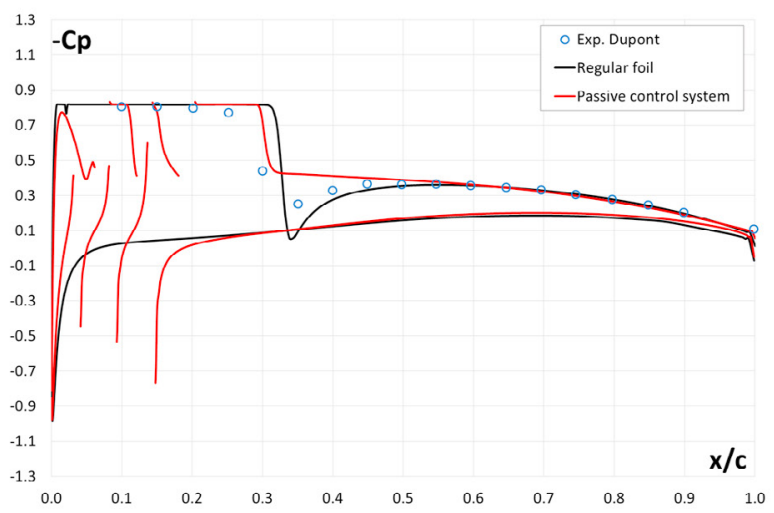

Fig. 5. Pressure coefficient on the suction and pressure side of the NACA0009 hydrofoil with $\alpha=2.5^{\circ}$ in fully wetted flow (a) and cavitating flow (b) conditions with $k-k l-\omega$ turbulence model. The results of the regular and modified geometry are compared with experimental data [11]. 


\subsection{Multi-phase flow}

The results show a good agreement between the pressure coefficient of the regular foil with $\alpha=2.5^{\circ}$ under cavitating flow conditions and the experimental results. The results are depicted in Figure 5b. Moreover, the curves of the modified geometry highlights that there are no large variation in either fully wetted and cavitating flow conditions (Figures 5a, 5b). To compare the performance of the original foil with and without the slots, at different angles of attack, the values of the $C_{L}, C_{D}$ and $C_{L} / C_{D}$ have been calculated. Moreover, the volumes occupied by the cavities have been calculated. The variations are resumed in Table 2 .

Table 2. Comparison of the hydrofoil performance at different angles of attack with and without passive cavitaion system control under multi-phase flow conditions.

\begin{tabular}{llllllllll}
\hline Cases & $\begin{array}{l}\text { Passive } \\
\text { control system }\end{array}$ & $\sigma$ & $C_{D}$ & $C_{L}$ & $C_{L} / C_{D}$ & var. $C_{D}[\%]$ & var. $C_{L}[\%]$ & var. $C_{L} / C_{D}[\%]$ & var. $V_{\text {cavity }}[\%]$ \\
\hline $0^{\circ}$ & no & 0.81 & 0.0204 & 0 & & & & \\
$0^{\circ}$ & yes & 0.81 & 0.0570 & 0.0642 & & 179 & & -39 & -93 \\
$2.5^{\circ}$ & no & 0.81 & 0.0248 & 0.3730 & 15.00 & & -35 & -35 & -54 \\
$2.5^{\circ}$ & yes & 0.81 & 0.0284 & 0.2404 & 9.15 & 12 & & -31 & \\
$5^{\circ}$ & no & 0.81 & 0.0496 & 0.6360 & 12.80 & & \\
$5^{\circ}$ & yes & 0.81 & 0.0622 & 0.4390 & 7.06 & 25 & & \\
\hline
\end{tabular}

The comparison of the mean liquid volume fraction, $\alpha_{l}$, of the regular foil against the modified geometry draw attention to the strong reduction of the cavitation phenomenon (Figure 6). This confirms the ability of the passive control system proposed in this work to suppress the cavitation inception at the leading edge of the hydrofoil, but also when cavitation largely affects the hydrofoil, like in the case of $5^{\circ}$, the system proposed is able to reduce the cavity to a significant extent. Indeed the slots are able to increase the pressure locally at the leading edge of the foil without promoting flow separation. Their effect is shown in Figure 6.

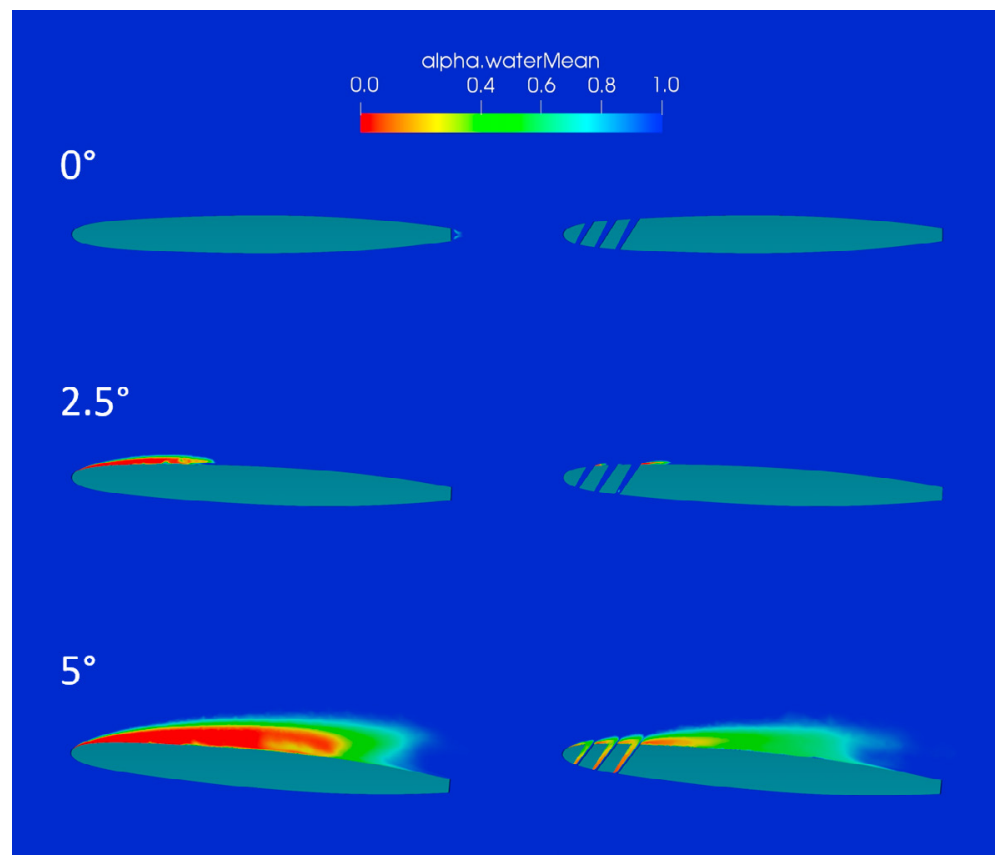

Fig. 6. Contours of the mean liquid volume fraction, $\alpha_{l}$, with and without the introduction of the passive cavitation control at different angles of attack. 


\section{Conclusions}

In this work, the performance of the NACA0009 hydrofoil with the introduction of geometry modifications working as a passive cavitation control system has been investigated with different angles of attack with the purpose to reproduce the leading edge of impeller vanes working at part loads. The study focuses on understanding how these modifications affect the regular performance of the system and conversely improve the performance when cavitation occurs by suppressing the cavity region. The passive cavitation control considers slots passing through the body of the foil that allow the pressure transmission from the pressure side to the suction side of the foil. The numerical analyses have been performed by solving 2D U-RANS equations with the mass transfer model proposed by Schnerr and Sauer [13] and the $k-k l-\omega$ turbulence model. The hydrofoils have been tested in single and multi-phase flow conditions and the results compared against the experimental data, when available $\left(2.5^{\circ}\right)$. The performance of the hydrofoil has been evaluated in terms of $C_{D}, C_{L}, C_{L} / C_{D}$ and vapour volume fraction $\left(\alpha_{v}\right)$. They show remarkable advantages, i.e., strong reduction of the vapour volume fraction (up to -93\%) even if accompanied by a loss of performance, $C_{L}$ reduction $(-31 \%)$ and $C_{D}$ increase $(+25 \%)$, respectively. This level of loss can be acceptable considering that the current case aims to reproduce the forward part of impeller vanes. Actually the impeller vane is longer than the foil analyzed here, therefore the losses will be localized at the leading edge whereas the benefit from suppressing the cavitation can improve the entire functionality of the pump channels. In the future work an optimization tool will be studied with the aim to design a symmetric configuration, which can be used when the hydraulic turbomachine works either at part or over loads.

\section{References}

[1] C.E. Brennen, "Cavitation and Bubble Dynamics, Oxford University Press" (1995).

[2] C.E. Brennen, "Fundamentals of Multiphase flows, Oxford University Press", (1995).

[3] A Cervone, C Bramanti, E Rapposelli, L dAgostino, (2006) ”Thermal cavitation experiments on a NACA 0015 hydrofoil.” J. Fluids Eng. 128 (2): 326331.

[4] M Dular, R Bachert, B Stoffel and B Åirok, (2004) "Experimental evaluation of numerical simulation of cavitating flow around hydrofoil" European Journal of Mechanics B/Fluids 24 (2005): 522538.

[5] S Barre, J Rolland, G Boitel, E Goncalves and R Fortes Patella, (2008) "Experiments and modeling of cavitating flows in venturi: attached sheet cavitation." European Journal of Mechanics B/Fluids

[6] B Stutz and JL Reboud, (1997) "Experiments one unsteady cavitation. ”Springer-Verlag Berlin Heidelberg. Exp. Fluids 22: 191198.

[7] T. Capurso, M. Lopez, M. Lorusso, M. Torresi, G. Pascazio, S.M. Camporeale, B. Fortunato, Numerical investigation of cavitation on a NACA0015 hydrofoil by means of OpenFOAM, In Energy Procedia, Volume 126, 2017, Pages 794-801, ISSN 1876-6102.

[8] Ichiro Kumagai, Yoshiaki Takahashi and Yuichi Murai 'Power-saving device for air bubble generation using a hydrofoil to reduce ship drag: Theory, experiments, and application to ships", Ocean Engineering (2015) doi.org/10.1016/j.oceaneng.2014.11.019.

[9] Jian Huang, Chao Yu, Yiwei Wang, Chang Xu and Chenguang Huang 'Passive control of cavitating flow around an axisymmetric projectile by using a trip bar", Theoretical and Applied Mechanics letters (2017) doi.org/10.1016/j.taml.2017.09.003.

[10] Weiguo Zhao, Guoshou Zhao, "An active method to control cavitation in a centrifugal pump by obstacles". Advances in mechanical engineering, (2017), doi.org/10.1177/1687814017732940.

[11] Dupont, P., 1991. Etude de la dynamique dune poche de cavitation partielle en vuede la prediction de lerosion dans les turbomachines hydrauliques. Ph.D. thesis, Ecole Polythechnique Federale de Lausanne, Lausanne, Switzerland.

[12] T. Capurso, S.M. Camporeale, B. Fortunato, M. Torresi, "Implementation of a passive control system for limiting cavitation around hydrofoils", 29th IAHR, Kyoto (Accepted).

[13] J. Sauer and G.H. Schnerr "Unsteady cavitating flow - a new cavitation model based on a modified front capturing method and bubble dynamics", In Fluids Engineering Summer Conference, Proceedings of FEDSM, (2000).

[14] J. Furst "Numerical simulation of transitional flows with laminar kinetic energy". Engineering Mechanics, 20(5):379388, (2013).

[15] Michel, J. P. Franc and J. M. Michel, "Fundamentals of Cavitation", (2005).

[16] Y. A. Bouziad, Phisical modelling of leading edge cavitation: computational methodologies and application to hydraulic machinery [Ph.D. thesis], Ecole Polytechnique Federale de Lausanne, 2005. 